\section{LA IMPORTANCIA DE LA CULTURA TECNOLÓGICA EN EL MOVIMIENTO MAKER}

\author{
Raúl Tabarés Gutiérrez \\ Fundación TECNALIA RESEARCH \& INNOVATION \\ ORCID iD: https://orcid.org/0000-0002-8149-3534 \\ raul.tabares@tecnalia.com
}

Cómo citar este artículo/Citation: Tabarés Gutiérrez, R., (2018). La importancia de la cultura tecnológica en el movimiento maker. Arbor, 194 (789): a471. https://doi.org/10.3989/ arbor.2018.789n3013

Recibido: 14 octubre 2017. Aceptado: 19 marzo 2018.

RESUMEN: La emergencia global del movimiento maker constituye uno de los fenómenos más recientes de difusión y apropiación de tecnologías por parte de la sociedad. El vencimiento de varias patentes en los campos de la fabricación digital y de la microelectrónica, junto con un reverdecimiento de la mentalidad DIY (Do It Yourself) y con la popularización de espacios, laboratorios y eventos temáticos por buena parte de la geografía internacional, han ayudado a consolidar una creciente presencia en la sociedad del enfoque conocido como aprender haciendo. En este artículo examinamos cómo ha surgido este movimiento e identificamos los principales valores que inspiran esta filosofía de capacitación de la ciudadanía a través de la tecnología. Así mismo analizamos las oportunidades que plantea este fenómeno para la promoción de una cultura tecnológica en la sociedad y también las contradicciones, mitos y retos que en él se encierran.

PALABRAS CLAVE: P2P; difusión social; apropiación social; STEM; cultura digital; movimiento maker; DIY.

\section{THE SIGNIFICANCE OF TECHNOLOGICAL CULTURE IN THE MAKER MOVEMENT}

Copyright: (C) 2018 CSIC. Este es un artículo de acceso abierto distribuido bajo los términos de la licencia de uso y distribución Creative Commons Reconocimiento 4.0 Internacional (CC BY 4.0).
ABSTRACT: The rising of the maker movement globally constitutes one of the most recent phenomena of social diffusion and social appropriation of technologies. The expiration of several patents in the fields of digital manufacturing and microelectronics in combination with the rebirth of a DIY (Do It Yourself) state of mind and the popularization of different spaces, laboratories and thematic events at international level have helped to consolidate a growing presence in society of this learning by doing approach. In this article we examine the emergence of this movement and identify the main values that lie behind this philosophy for empowering citizenship through technology. Moreover, we analyze the opportunities presented by this phenomenon for promoting a technological culture in society and the contradictions, myths and challenges surrounding it.

KEYWORDS: P2P; social diffusion; social appropriation; STEM; digital culture; maker movement; DIY. 


\section{INTRODUCCIÓN}

Durante los últimos años hemos asistido a una popularización del movimiento maker (Dougherty, 2012). Este fenómeno ha surgido a escala global por la aparición de lo que se conoce como open hardware, que ha permitido el surgimiento de innovaciones con diseño abierto y de bajo coste. El gran desarrollo de nuevas tecnologías de libre acceso en torno a la fabricación digital (Birtchnell y Urry, 2013) ha creado nuevas y numerosas posibilidades para la ciudadanía, de cara a adoptar los beneficios que promete la producción personalizada (Ratto y Ree, 2012).

Este factor, unido al surgimiento de espacios orientados a la colaboración como Fab Labs, Makerspaces o Media Labs, donde se trabaja en diversos proyectos en un régimen de producción social y con una marcada orientación hacia necesidades ciudadanas, ha permitido el desarrollo de nuevas formas de innovación colaborativa basadas en la producción social de tecnologías no propietarias. Por ello, el movimiento maker está atrayendo la atención de todo tipo de instituciones interesadas en las posibilidades que encierra este fenómeno para la educación, la ciencia, la tecnología y la sostenibilidad.

Con el objetivo de explorar las oportunidades, amenazas y mitos que encierra este fenómeno repasamos sus orígenes y sus interrelaciones con la contracultura norteamericana y con la cultura hacker (Himanen, 2002). También analizamos las visiones que promueve esta corriente respecto a la cultura tecnológica incorporada y a la cultura tecnológica no incorporada, con el fin de diseccionar la dimensión axiológica de este paradigma tecno-social.

\section{LOS ORÍGENES}

A pesar de que los comienzos del movimiento maker pueden identificarse claramente con una serie de tecnologías no propietarias que son producto de la expiración de una serie de patentes en campos como la microelectrónica, la impresión 3D o el diseño 3D, hay que añadir que además de estas nuevas tecnologías libres hay otro tipo de factores sociales que han propiciado su irrupción en la sociedad. Así, nuevas formas de trabajo como la producción social (Benkler, 2006) colaborativa o lo que se conoce en inglés como Peer to Peer o P2 $\mathrm{P}^{1}$ y un énfasis en el aprendizaje informal y no formal, que se producen de manera online y off-line (Tabarés-Gutiérrez, 2016) han contribuido claramente a su consolidación. Conviene aclarar que el movimiento maker hunde sus raíces en otros fenómenos anteriores de apropiación social de la tecnología como el Do It Yourself (DIY) y la ética hacker. El Do It Yourself es una corriente de pensamiento bastante madura que hunde sus orígenes hasta los años '20, con manifestaciones bastante populares como las emisiones pirata de radio (Haring, 2008). Esta filosofía de hacer las cosas por uno mismo ha estado más o menos presente desde los años '70 (Kuznetsov y Paulos, 2010) y ha vivido épocas muy populares como en las décadas de los '80 y los '90 (Atkinson, 2006), o como la que vivimos actualmente, en la cual ha habido un resurgir de este enfoque gracias a la expiración de diversas patentes en el campo de la fabricación digital y de la microelectrónica (Fox, 2014). Para entender el movimiento DIY deberíamos primero definir en qué consiste exactamente, lo cual no es fácil, ya que comprimir las tres grandes áreas (diseño, arte y artesanía) en las que normalmente se divide no es simple (Atkinson, 2006). Una definición bastante útil es la que proponen los autores Kuznetsov y Paulos, los cuales definen el fenómeno DIY como "cualquier creación, modificación o reparación de objetos sin la ayuda de profesionales remunerados" (Kuznetsov y Paulos, 2010, p. 1).

Tras esta definición podemos intuir que esta filosofía encierra un componente de altruismo y de actitud amateur, ya que el énfasis de la misma se centra en la falta de ayuda o soporte profesional para el desarrollo de tareas orientadas a la producción o a la modificación de objetos diversos con un fin determinado. Este interés por el desarrollo de creaciones y modificaciones que carezcan de una supervisión profesional es el que posteriormente ha dado lugar a la ética hacker (Himanen, 2002) y al que nos ocupa en este texto, el movimiento maker (Dougherty, 2012). Fenómenos que también podemos relacionar con la contracultura americana del siglo XX y que también se encuentran entre las influencias de los grandes pioneros del software y del hardware comercial, los cuales crearon una nueva industria tecnológica con unos valores diferentes a los que había en otras culturas ingenieriles (Barbrook y Cameron, 1996). Tanto el movimiento maker como la filosofía DIY comparten esa querencia por la creación y modificación de objetos físicos o digitales pero, al mismo tiempo, la cultura maker también se diferencia de la primigenia filosofía DIY en el uso de tecnologías libres, en su producción social (Benkler, 2006) y en el acceso a Internet como fuente de conocimiento, discusión, co-creación y promoción de sus ideas (TabarésGutiérrez, 2016). Este tipo de súper usuarios de la red se han vuelto cada vez más comunes y populares gracias a las nuevas posibilidades de producción personal 
de artefactos que ha introducido el paradigma de la fabricación personalizada y de la microelectrónica, además de otro tipo de tecnologías de diseño abierto que se conocen como open hardware.

Para ayudar a entender las diferencias del movimiento maker con otras contraculturas tecnológicas nos gustaría citar el trabajo de Silvia Lindtner, la cual ha publicado extensamente sobre la cultura maker y a la cual reconoce ciertas diferencias con la anterior cultura hacker (Himanen, 2002), que se caracterizaba por un énfasis en el código; "Ia cultura maker contemporánea no sólo se preocupa por las tecnologías abiertas de Internet y lo digital, sino también de objetos físicos como diseños de artefactos, sensores y conectores de red que unen el mundo digital con el físico. Mientras la cultura hacker anterior se preocupaba por el código y el funcionamiento de Internet, esta nueva cultura maker se preocupa por los diseños del hardware y el funcionamiento de las tecnologías que hacen posible la Internet de las Cosas" (Lindtner, 2014).

Como relata esta autora la nueva cultura maker también se caracteriza por involucrar a individuos que intentan jugar y experimentar con la tecnología al igual que los hackers (Lindtner, 2015), pero cuyo ámbito de experimentación es mucho mayor debido al desarrollo de lo que se conoce como open hardware, que ha permitido enlazar el mundo físico con el virtual a través de artefactos que poseen un diseño no propietario (Gershenfeld, 2005). Es el caso de iniciativas tan exitosas como Arduino (Dafermos, 2015), Raspberry Pi, Makerbot (Ratto y Ree, 2012) o RepRap (Kostakis y Papachristou, 2014), que han permitido a su vez el desarrollo de un sinfín de proyectos de bajo coste pero con un gran impacto y popularidad en Internet. Estas tecnologías funcionan como plataformas de código libre que permiten desarrollar a multitud de aficionados y entusiastas de la tecnología diversos artefactos en el ámbito de la microelectrónica, las telecomunicaciones o la fabricación digital. Iniciativas que normalmente son de bajo coste pero que se orientan a necesidades específicas de diversos colectivos marginales de la sociedad y que no suelen estar cubiertas por la producción en masa. Es el caso de llaves de reemplazo para coches, mandos y joysticks diversos, sensores de presencia, herramientas para la monitorización ambiental, prótesis, ortesis, robots educativos y un largo etcétera. La gran ventaja que ofrecen estas soluciones de bajo coste es que no existe la necesidad de fabricar una gran tirada, con el elevado coste asociado que ello conlleva, sino que se pueden fabricar pequeños prototipos que pueden ser mejorados sin tener que fabricar de nuevo una serie más larga. Lo cual hace que se pueda desarrollar un mejor control y modificación sobre las diversas innovaciones, de manera que se adapten a las necesidades personales de sus usuarios en vez de lo contrario, que suele ser lo imperante en las estructuras de producción actuales.

Esta irrupción de la fabricación personalizada (Lipson y Kurman, 2010; Mota, 2011) y la micro-electrónica de bajo coste ha propiciado un gran interés en la ciudadanía en general y ello ha propiciado que espacios como Hackerspaces, Makerspaces o Fab Labs se hayan convertido en algo común en las ciudades. En estos lugares se celebran eventos temáticos, cursos y actividades de todo tipo orientadas a la producción colaborativa de objetos digitales (Smith, Hielscher, Dickel, Söderberg y Oost, 2013) capaces de cubrir diferentes necesidades sociales propuestas por la ciudadanía. Es por ello por lo que el fenómeno se abre paso en el entorno principalmente urbano, pero no solo en él, ya que hay notables experiencias en el mundo rural y en nuestro país tenemos un buen ejemplo como es el caso de la experiencia que se lleva a cabo en Calafou ${ }^{2}$. Además, también estamos asistiendo a un creciente desarrollo de tecnologías libres para la industria agrícola y prueba de ello es la iniciativa Farmbot (Cruz, Herrington y Rodríguez, 2014), la cual nos muestra cómo las posibilidades del movimiento maker pueden tener un gran recorrido en el entorno rural, debido a sus bajos costes y a su posibilidad de aplicación directa a diversas problemáticas concretas que afectan a este medio.

Todos estos espacios que hemos citado anteriormente se caracterizan por disponer de un equipamiento en el cual podemos listar varios tipos de máquinas tales como impresoras 3D, escáneres en 3D, kits de soldadura, cortadoras láser, CNC's y una larga lista de herramientas que facilitan el desarrollo colaborativo de proyectos de fabricación a través de los cuales poder aprender y experimentar con la tecnología. Además, el desarrollo en la Web de plataformas como Thingiverse ${ }^{3}$ y otras muchas más que funcionan como artefactos de conocimiento (Locoro, Ravarini, Cabitza y Mari, 2017) permite a estos usuarios descargar diseños, instrucciones y tutoriales para replicar proyectos ya realizados por otros usuarios en otros laboratorios alrededor del mundo o compartir sus propios diseños a través de este tipo de plataformas. Además, estas plataformas también constituyen un ágora pública donde poder discutir con diversos usuarios y creadores de los proyectos las posibles dudas o preguntas que surgen en los procesos de fabricación y desarrollo tecnológico. 
Debido a la variedad de los proyectos que se han desarrollado en estos espacios maker y al impacto que han tenido muchos de ellos fuera del entorno en el que han sido creados, algunos autores y la prensa en general han comenzado a hablar de "una nueva revolución industrial" (Anderson, 2012; A third industrial revolution) o "una democratización de la fabricación" (Mota, 2011). Si bien es cierto que este movimiento provee de herramientas para favorecer una producción más social y fomentar la innovación y el emprendimiento en la ciudadanía (Gershenfeld, 2005; Smith et al., 2013), no por ello hay que caer en ciertas visiones tecno-utópicas que se alimentan desde los medios de comunicación (Sivek, 2011). En este sentido, es innegable que este tipo de espacios y dinámicas proveen de innumerables oportunidades para la ciudadanía a la hora de volver a reconectarla con las actividades de fabricación que han sido desterradas del centro de las ciudades y de muchos de los países con economías avanzadas, a través de la deslocalización de centros productivos hacia países en vías de desarrollo con unos menores costes laborales. Al mismo tiempo, también debemos resaltar que el movimiento maker ofrece oportunidades para promover modalidades de producción y consumo más sostenibles, pero no por ello debemos olvidar que las principales barreras en el uso de este tipo de tecnologías libres en las que se fundamente esta nueva ola de hazlo tú mismo no residen en el coste, sino en el acceso al conocimiento y su desarrollo (Morozov, 2014, 23 de enero). Precisamente este se ha vuelto cada vez más fragmentado y disperso gracias a la pléyade de plataformas (Locoro et al., 2017) y entornos colaborativos que han surgido a partir de la explosión de la Web 2.0 (Tabarés-Gutiérrez, 2015, 29 de julio). Además, su profusión y creciente especialización provoca numerosas brechas digitales debido al surgimiento de diversas tecnologías que se han desarrollado gracias a la digitalización de sectores ya establecidos, como el de la fabricación, que ahora son mediados a través de plataformas y herramientas digitales.

\section{POPULARIZACIÓN DE LA CULTURA MAKER}

\section{Espacios y laboratorios}

La popularización del movimiento maker se ha producido a escala internacional gracias al efecto red (Farrell y Klemperer, 2007) que se produce en Internet y al papel que desempeñan las comunidades on-line en el espacio virtual (Rheingold, 1996), pero también a través de la difusión de innumerables espacios físicos donde se realizan actividades orientadas a interactuar con este tipo de tecnologías no propietarias y de bajo coste (Niaros, Kostakis y Drechsler, 2017). Este es el caso de espacios como Fab Labs, Makerspaces y Hackerspaces, los cuales permiten a sus usuarios desarrollar proyectos colaborativos de fabricación digital o acceder a cursos, seminarios y jornadas donde se puede aprender a dominar las máquinas indicadas y adquirir los conocimientos necesarios para desarroIlar las capacidades adecuadas. Algunos de estos espacios pertenecen a redes más formales como es el caso de los Fab Labs, los cuales surgen a principios del año 2000 a partir de la iniciativa de Neil Gershenfeld que por aquel entonces era director del Center for Bits and Atoms (CBA), en el Massachusetts Institute of Technology (MIT). El origen de esta red se debe a la financiación que recibe el CBA por parte de la National Science Foundation (NSF) y a la colaboración que se inicia con el Grasroots Invention Group, lo cual les permite adquirir una serie de máquinas que permiten "fabricarlo casi todo" (Gershenfeld, 2005). A partir de la primera fundación de este laboratorio se desarroIlan otros en diversos países del mundo, como la India o Noruega, en torno a 2002. A la creación de estos espacios le sigue el desarrollo de varios materiales didácticos que el propio Neil desarrolla para dar a conocer el funcionamiento de las máquinas que componen un Fab Lab e introducir los conceptos de fabricación digital y open hardware. Estos materiales son los que posteriormente formarán parte de lo que se conoce como Fab Academy: un programa de formación a distancia para instruir y certificar a las personas que lo deseen en las herramientas que se disponen en estos espacios de fabricación alternativos ${ }^{4}$.

Otras iniciativas como los Makerspaces o Hackerspaces no han conllevado el establecimiento de una red formal como la Fab Lab Network, ya que este tipo de espacios deben disponer de una serie de equipamiento específico para poder pertenecer formalmente a la red, pero también han experimentado un crecimiento notable durante los últimos años a través de redes informales. Este tipo de laboratorios urbanos también disponen de una serie de máquinas y herramientas de fabricación digital, además de desarrollar diversas actividades formativas y de contar con personas formadas en el uso de estas herramientas de fabricación digital (muchas de las cuales suelen haber realizado el curso del Fab Academy). En este tipo de laboratorios se suelen utilizar metodologías de aprendizaje cooperativo, a través del desarrollo de proyectos o retos que implican el aprendizaje multidisciplinar de diversas competencias y que se apoyan a su vez en el uso de software y de hardware libre. La popularización de 
Fab Labs, Makerspaces, Hackerspaces y otro tipo de laboratorios urbanos ha sido manifiesta durante los últimos años, no en vano el número de estos espacios no hace más que crecer continuamente. Así, la plataforma de fablabs.io muestra cómo hay unos 1.150 laboratorios activos ${ }^{5}$ en diversas partes del mundo y números similares se pueden encontrar en otros espacios de fabricación digital no adscritos a la red del MIT, ya que en la wiki colaborativa de Hackerspaces el número llega hasta los 1.355 laboratorios activos ${ }^{6}$.

Otro modelo interesante que merece atención es el sistema de franquicias estadounidense TechShop ${ }^{7}$, que ofrece servicios de membrecía a sus usuarios para acceder a un equipamiento más profesional del que se puede encontrar en los anteriores laboratorios. La principal fortaleza de este modelo radica en sus nexos con la industria (acuerdos con Ford o Autodesk en EE. UU., Samsung o Fujitsu en Japón y Leroy Merlin en Francia), ya que facilita el escalado industrial de los prototipos. Lamentablemente esta empresa anunció recientemente su quiebra en EE. UU. (pero no en otros países), lo cual es un síntoma claro de la exigente búsqueda de modelos de negocio sostenibles que todos estos espacios deben asumir para garantizar su supervivencia ${ }^{8}$.

Por último, las Maker Faires o ferias maker son los eventos más populares donde se pueden contemplar las creaciones realizadas por multitud de apasionados de la tecnología. Este tipo de exposiciones surgen en 2006 gracias al empuje de la revista Make (Sivek, 2011) para "celebrar el movimiento maker"9 y dar visibilidad a todos esos proyectos que han sido concebidos bajo la filosofía DIY y que han sido desarrollados en garajes o locales de barrio. A lo largo de los últimos años este tipo de ferias han atraído una gran asistencia de público y se han consolidado en multitud de localizaciones por todo el planeta. En nuestro país se han desarrollado eventos de este tipo en ciudades como Barcelona, Bilbao, León, Madrid o Santiago de Compostela. Dichos eventos permiten visualizar el potencial de las creaciones que se desarrollan en un régimen de producción colaborativa y social, al mismo tiempo que intentan atraer el interés de otro tipo de organizaciones o instituciones más formales tales como empresas, ayuntamientos o agencias de desarrollo, que no suelen estar en contacto con este tipo de comunidades de innovación.

\section{Apoyos institucionales}

La consolidación de este movimiento en la sociedad ha atraído la atención de diversas instituciones que han decidido tender puentes con este fenómeno.
Quizás el caso de EE. UU. haya sido uno de los más mediáticos, ya que la administración Obama decidió celebrar una Maker Faire dentro de la propia Casa Blanca en 2014 bajo el lema White House Maker Fai$r e^{10}$. Dicho evento se enmarcó dentro de la semana de actividades dedicadas al movimiento maker, conocida como la National Week of Making ${ }^{11}$, en la que suelen participar activamente universidades, colegios, ayuntamientos y empresas. Además, el gobierno norteamericano también ha realizado diversas acciones de difusión y sensibilización para impulsar la cultura maker en diferentes ámbitos. Entre estas acciones destaca la iniciativa Maker Cities, la cual consiste en una alianza de más de 100 ciudades estadounidenses que se han comprometido a apoyar el desarrollo de espacios maker, a promover eventos relacionados con el fenómeno y a apoyar acciones que provengan de este tipo de colectivos ${ }^{12}$.

Otro de los países que también ha impulsado de manera notable el desarrollo de este tipo de actividades es China. En 2010 y en Shanghái, se inauguró el primer espacio dedicado a la cultura maker denominado XinCheJian (traducido como nuevo taller o nueva fábrica) y al año siguiente la propia ciudad apoyó la apertura de 100 espacios más para promover los valores de la cultura maker (Lindtner y Li, 2012). Otras ciudades como Nanjing, Pekín, Hangzhou y Shenzhen también albergan este tipo de espacios y han apoyado diversas iniciativas relacionadas con la promoción de estos laboratorios de fabricación digital. Especialmente representativo es el caso de Shenzhen, ya que este pequeño pueblo pesquero ha pasado a convertirse en un escaso periodo de tiempo en la capital tecnológica del gigante asiático y en uno de los centros neurálgicos de la fabricación a nivel mundial. Compañías como Foxconn, que cuentan con cientos de miles de empleados y ensamblan productos para todo tipo de gigantes tecnológicos como Apple, Sony o Amazon, tienen su sede en esta ciudad. Precisamente estas empresas que se dedican al desarrollo de alta tecnología como Foxconn o Xiaomi han adoptado prácticas propias del movimiento maker para mejorar su competitividad, tales como el establecimiento de redes informales para compartir diseños de hardware, establecer listas de materiales y otras (Lindtner, 2015). Este tipo de experiencias se ven motivadas por razones económicas y no por factores culturales, como pudiéramos pensar inicialmente (Lindtner, 2014), ya que el fenómeno de una producción open-source ha estado de manera más o menos presente en la historia de este país desde los inicios de su industrialización masiva, con el objetivo de abaratar costes y fomentar un ecosistema de innovación (Lindtner, 2015; Lindtner y Li, 2012). 
En lo que se refiere al continente europeo podemos observar varias iniciativas por parte de la Comisión Europea para apoyar este tipo de espacios y organizaciones. A través del programa marco de investigación Horizonte 2020 existen varios sub-programas de trabajo específicos como Science with and for Society (SwafS) ${ }^{13}$ que tratan de fomentar la educación científico-tecnológica de la ciudadanía y de los escolares a través de la financiación de proyectos colaborativos de investigación que promuevan el enfoque STEM (Science, Technology, Engineering and Mathematics). Este término fue acuñado por la NSF norteamericana en los años '90 para subrayar la importancia de trabajar en la enseñanza de estas disciplinas de manera integrada desde una edad temprana y orientarlas hacia la resolución de problemas concretos. Este enfoque educativo se caracteriza por enseñar de forma conjunta Ciencias, Matemáticas y Tecnología a través de la realización de proyectos en equipo que involucran a los estudiantes en problemas auténticos para los que se necesitan soluciones reales, tal y como ocurre en la ingeniería. Al mismo tiempo, la Comisión Europea ha financiado directamente varios proyectos que im- pulsan la investigación en torno a este tipo de espacios de fabricación como Make-IT ${ }^{14}$, Making Sense ${ }^{15}$ u OPENMAKER ${ }^{16}$. Otros programas de investigación como Erasmust auspiciados por la Comunidad Europea también han hecho lo propio a través de proyectos como $O D \& M^{17}$. La Comunidad Europea también ha dedicado diversos esfuerzos a la realización de eventos específicos orientados a la exaltación de la cultura maker y por ello ha apoyado la celebración de la Maker Faire en Roma ${ }^{18}$ como el evento de referencia del movimiento maker en el continente. Por último, también ha promovido su apoyo institucional a otro tipo de eventos más relacionados con la cultura del emprendimiento y en los que ha habido apoyo por parte de grandes empresas relacionadas con las tecnologías digitales como Makers Town ${ }^{19}$.

En lo que se refiere a nuestro país nos gustaría mencionar dos iniciativas singulares. En este sentido tenemos que reseñar la experiencia que lleva a cabo desde hace algunos años el Ayuntamiento de Barcelona a través de la red de Ateneus de Fabricació ${ }^{20}$. Esta red de espacios de fabricación digital ha contribuido a acercar el potencial de este tipo de tecnologías emergen-

Figura 1. El evento Makers Town, celebrado con el apoyo de la Comisión Europea en Bruselas

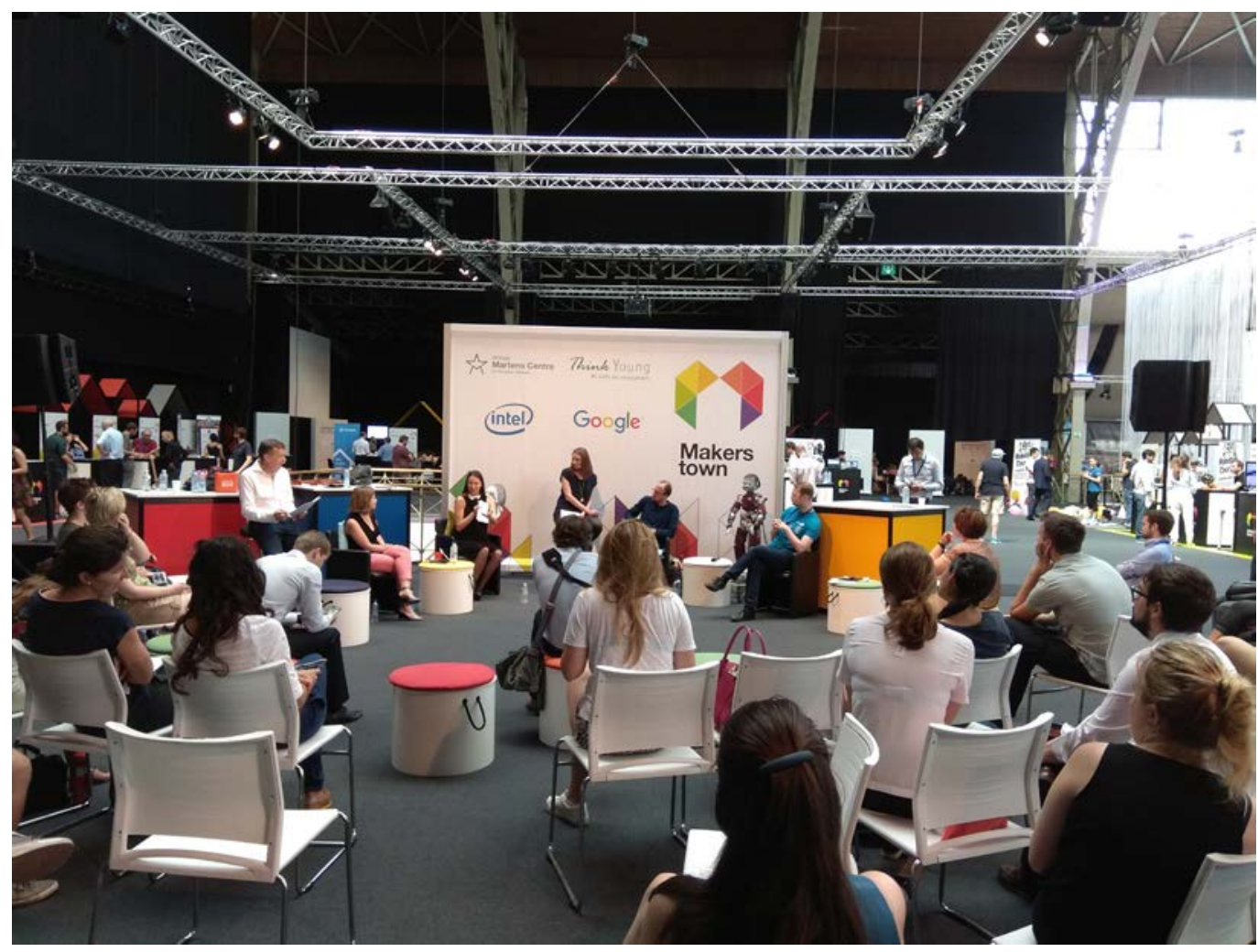

Fuente: Fotografía propia. 
tes hasta diversos barrios de la ciudad, con el objetivo de establecer espacios de conocimiento y al mismo tiempo de acción ciudadana y en los que "desde niños hasta jubilados puedan entrar en el mundo de la fabricación digital"21. Otra experiencia que también destacamos es la que apoya la Viceconsejería de Formación Profesional (FP) del Gobierno Vasco a través de la red Ikaslab ${ }^{22}$. Una red de espacios dedicados a la impresión en 3D en diversos centros de FP de la comunidad autónoma, con el objetivo de familiarizar a los estudiantes del sistema con esta tecnología y ampliar sus conocimientos, capacidades y habilidades para mejorar su futura empleabilidad de cara a las empresas. Esta iniciativa se enmarca en la ambiciosa estrategia de Industria $4.0^{23}$ que dispone el gobierno vasco para apoyar la digitalización del sector de la fabricación.

\section{CULTURA TECNOLÓGICA}

Como hemos visto, el movimiento maker va más allá de un colectivo con grandes habilidades digitales para desarrollar proyectos colaborativos. Encontramos una fuerte carga ideológica en la que se resaltan valores como la cooperación, la producción social entre iguales, el acceso al conocimiento, la promoción de la innovación y el rechazo de la propiedad intelectual, entre otros. Este ideario ha llevado a algunos autores a preguntarse si estos nuevos innovadores sociales representan una nueva etapa en la lucha de clases (Dafermos y Söderberg, 2009), ya que como podemos observar se pone de relevancia la importancia del "aprender haciendo" de cara al empoderamiento tecnológico de la ciudadanía. Creemos por tanto que es necesario analizar los valores que se promueven en este tipo de cultura tecnológica con el objetivo de diseccionar el contenido ideológico de la misma y así poder entender el sentido que se promueve.

Llegados a este punto nos gustaría introducir y definir el concepto de cultura tecnológica de un grupo social y para ello prestamos atención a la definición de Miguel Ángel Quintanilla (2005), quien la define como "Ia información representacional, práctica o valorativa que comparten los miembros del grupo y que son potencialmente relevantes para la creación, producción, posesión o utilización de tecnologías o sistemas tecnológicos" (Quintanilla, 2005, p. 255).

Esta definición de Quintanilla se afianza en otras contribuciones que el mismo autor desarrolla previamente (Quintanilla, 1998) y que se basan a su vez en la definición que Jesús Mosterín propone de cultura: "la información transmitida por aprendizaje social entre animales de la misma especie" (Mosterín, 1993).
Quitanilla (2005) establece tres componentes de la cultura tecnológica:

- La información representacional, como conocimientos, creencias y representaciones conceptuales o simbólicas de las técnicas.

- La información práctica, que incluye hábitos, reglas de comportamiento y habilidades técnicas específicas.

- La información valorativa, en la que se incluyen fines, valores y actitudes relativos al diseño, uso, etc. de sistemas técnicos y de conocimientos técnicos.

Estos tres componentes de la cultura tecnológica que citamos aquí se pueden presentar en dos modalidades: aquellos que están incorporados a sistemas técnicos y aquellos otros que no lo están, a pesar de ser parte de la cultura técnica de un grupo social. Así, podemos hablar de cultura técnica en sentido estricto y de cultura técnica en sentido lato (Quintanilla, 1998). Hablamos por lo tanto de cultura técnica incorporada como "Ia información cultural (representacional, práctica o valorativa) que el agente u operador del sistema debe tener para que el sistema funcione adecuadamente" (Quintanilla, 2005, p. 256) y de cultura técnica no incorporada "cuando se trata de elementos culturales relacionados con el uso, producción, etc. de sistemas técnicos, pero que no son esenciales para el correcto funcionamiento de éstos" (Quintanilla, 2005, p. 256).

Así mismo debemos aclarar que las fronteras entre estos dos tipos de culturas no es algo rígido, ya que el desarrollo y difusión de las tecnologías propicia la adquisición de contenidos culturales por parte de los sistemas técnicos y estos a su vez generan nuevos rasgos técnico-culturales en sentido lato (Quintanilla, 1998). Este precisamente es el caso que nos ocupa, ya que podemos observar claramente cómo la fuerte carga ideológica que presenta el movimiento maker propicia unos rasgos de cultura tecnológica no incorporada que determinan el uso y disfrute de las tecnologías libres que se utilizan. La dimensión axiológica o valorativa de la cultura tecnológica se pone de relieve a la hora de utilizar las tecnologías open source con las que el fenómeno ha ido creciendo. Esto ha cristalizado de forma reveladora en el desarrollo de varios manifiestos que exaltan la ética y los valores de esta filosofía DIY tanto a nivel internacional (Hatch, 2013) como nacional (García-Sáez, 2016).

Como es bien sabido, los factores culturales tienen una gran influencia sobre los procesos de innovación (Echeverría, 2008; Quintanilla, 1997; Toboso-Martín, 2014) y por ello también prestamos atención a la 
propuesta que hace Quintanilla para calcular el nivel de innovación de un grupo social (empresa, país, región, etc.), que articula en torno a dos factores (Quintanilla, 2005):

- La capacidad de innovación tecnológica, la cual depende de las tecnologías que estén disponibles para un determinado grupo social. Es decir, cultura tecnológica incorporada a sistemas técnicos que está integrada en la cultura del grupo.

- La propensión a innovar en el ámbito de la tecnología que tiene un determinado grupo social y que depende de representaciones, actitudes, valores y pautas de comportamiento referidas a la tecnología y a los sistemas técnicos que comparten los miembros del grupo. Es decir, cultura tecnológica no incorporada.

Como el propio autor indica, estas dos hipótesis se pueden resumir en que "no basta con poder innovar, es preciso además querer" (Quintanilla, 2005). Es decir, la primera de las hipótesis plantea el acceso a tecnologías con las cuales se puedan desarrollar innovaciones, y la segunda se enmarca en factores culturales que puedan facilitar una cultura de la innovación tales como la disposición a asumir riesgos, la valoración de la creatividad, la búsqueda de la perfección en el trabajo, la confianza en la ciencia y la tecnología como medio para resolver problemas, etc.

Creemos que esta propuesta de Quintanilla es más que relevante para el caso que nos ocupa ya que los elementos de cultura tecnológica axiológica no incorporada del movimiento maker apuntan precisamente en esta dirección. En otras palabras, promueven ese sentido de confianza en la tecnología como medio para poder resolver problemas, estimulan la creatividad y el valor del trabajo, a la vez que desmitifican el fracaso o el error, y lo hacen a través de un sentido colectivo de aprendizaje y pertenencia que se fomenta a través de diversas comunidades de práctica (Wenger, 1999). Podemos hablar de que fomentan una cultura proclive a la innovación, al aprendizaje continuo, a la difusión del conocimiento de manera libre, al trabajo cooperativo y a otra serie de factores que se consideran primordiales de cara a la transición hacia una sociedad de la innovación y el conocimiento (Olivé, 2005).

Por otro lado, el equipamiento tecnológico con el que se suele dotar a este tipo de espacios ha permitido que los usuarios de los mismos hayan podido desarrollar una cultura tecnológica incorporada al tener acceso a una serie de tecnologías de fabricación digital que de otra forma sería muy difícil desarrollar. Pode- mos concluir por tanto que estos espacios han contribuido claramente a la difusión social (Rogers, 1962) y a la apropiación social (Toboso-Martín, 2014) de las tecnologías de fabricación digital, pero al mismo tiempo a la promoción de una cultura proclive a la innovación y además crítica con el desarrollo tecnológico. Quizás este sea el hecho más reseñable de este tipo de iniciativas ya que, si bien hemos comentado que este tipo de tecnologías se han popularizado por la expiración de una serie de patentes que han permitido su libre uso, quizás la mayor contribución del movimiento maker haya sido desde una dimensión axiológica que favorece el desarrollo de la innovación por parte de actores no habituales, lo cual constituye una innovación social (Echeverría, 2008) y al mismo tiempo produce vías alternativas al desarrollo tecnológico. En este sentido podemos hablar de que se ha producido una socialización de la I+D o una popularización de los procesos de innovación en la fabricación que ha conllevado el giro hacia una fabricación social (Tabarés-Gutiérrez, 2016; Tabarés-Gutiérrez, Sopelana, García y MorenoValdés, 2016), ya que estos espacios poseen unos recursos que permiten desplegar una cultura tecnológica incorporada a través de sus equipamientos, talleres, cursos y demás, pero al mismo tiempo fomentan una cultura tecnológica no incorporada que promueve la creatividad, el emprendimiento y la innovación.

\section{HORIZONTES DEL MOVIMIENTO MAKER}

\section{Oportunidades, mitos y retos}

El movimiento maker presenta un gran potencial de cara a promover una cultura de la innovación en la sociedad debido a la dimensión axiológica que conlleva, ya que las dinámicas de innovación abierta (Chesbrough, 2003; Von Hippel, 2005) que se establecen en los diversos espacios on-line y off-line que componen estas comunidades de innovadores sociales ofrecen numerosas oportunidades a la hora de involucrar a la ciudadanía con la tecnología y la fabricación. Por ello, el potencial de este tipo de espacios a la hora de fomentar procesos de co-creación entre diversos actores es destacable, debido a que los desarrollos tecnológicos que se producen en estas comunidades son resultado de las interacciones e iteraciones entre los diferentes grupos que se encuentran en estos espacios.

En este sentido quizás el sector educativo haya sido uno de los que más atención ha prestado a las posibilidades que plantea la cultura maker a la hora de repensar los métodos de aprendizaje actuales (Martin, 2015). Las narrativas de poder que acompañan a la 
producción de artefactos a través del uso de tecnologías de fabricación digital (Halverson y Sheridan, 2014) constituyen un elemento que facilita el empoderamiento personal a través del uso de la tecnología, el desarrollo de habilidades y competencias específicas y una mayor motivación e involucración hacia la consecución de los objetivos curriculares delimitados en su aprendizaje. Quizás el peligro de la incorporación de este tipo de tecnologías y espacios a las aulas radica más en adoptar un enfoque tecnocentrista y simplista, en vez de uno axiológico y holístico.

Aparte de la educación, la industria de la fabricación también puede beneficiarse de la cultura maker a la hora de impulsar una mayor sostenibilidad de las prácticas de producción y consumo (Kostakis, Niaros, Dafermos y Bauwens, 2015), además de adaptarse a las necesidades que demanda la digitalización de la industria. La irrupción de una fabricación abierta (Tabarés-Gutiérrez et al., 2018) propiciada por la combinación de tecnologías libres, la producción social de artefactos y la generación de conocimiento en red, representa también una oportunidad reseñable para las pymes industriales que muchas veces no disponen de los recursos necesarios para adquirir equipamiento de alta tecnología o contratar profesionales altamente cualificados. En este sentido, la facilidad de acceso a los espacios maker, la ausencia de licencias restrictivas en el uso de las tecnologías y el libre acceso a la información que se producen en este fenómeno pueden ayudar a este tipo de empresas a adoptar dinámicas que les permitan adaptarse al cada vez más acelerado ritmo de innovación tecnológica propiciado por la digitalización.

Por otro lado, también es necesario afrontar las visiones tecno-optimistas que normalmente se exponen en los medios de la cultura maker y las oportunidades que presenta (Sivek, 2011). Hay que resaltar que existen diversos retos y desafíos que se deben superar para poder hablar de un movimiento verdaderamente inclusivo. Entre ellos destaca el mismo "arquetipo de maker" ya que, según un estudio de Maker Media, ocho de cada diez makers son hombres, su media de edad es de 44 años y sus ingresos familiares rondan los $106.000 \$$. Además el $97 \%$ poseen una carrera universitaria y el $80 \%$ ha recibido alguna formación de tercer grado (Leonard, 2013, 26 de julio). Las cifras en Reino Unido y China son bastante similares con un $80 \%$ y un $77 \%$ de varones que se autodefinen como makers (Niaros et al., 2017). A pesar de que estos espacios de fabricación digital se han desarrollado en un considerable y diverso número de localizaciones, estos números nos hacen preguntarnos si no se están cometiendo los mismos errores que en otros modelos que fomentan la ciencia, la tecnología y la innovación o si simplemente son un reflejo de las desigualdades que se proyectan a través de modelos culturales sesgados y tan ampliamente difundidos como el de Silicon Valley. Los datos que existen nos indican que el movimiento maker está formado por individuos que podrían considerarse como élites educativas, ya que los conocimientos necesarios para utilizar este tipo de tecnologías siguen siendo altamente especializados. Por ello, podemos afirmar que las barreras de entrada al uso de la tecnología se han relajado pero no podemos decir lo mismo de las barreras referentes al acceso y uso del conocimiento (Morozov, 2014, 23 de enero), el cual podemos entrever como más fragmentado y especializado debido a la popularización de las plataformas digitales.

Al mismo tiempo también debemos desmitificar la explosión de creatividad y artesanía que a veces se asocia con este fenómeno (Kneese, Rosenblat y Boyd, 2014), ya que el hecho de que se compartan diseños en diversas plataformas on-line no implica que se favorezca la creación de nuevos modelos. En este sentido podemos encontrar un buen número de diseños para imprimir en 3D, diseños de placas de microelectrónica y otros contenidos que en multitud de ocasiones se replican en otra parte del mundo o se modifican parcialmente. No siempre este tipo de plataformas alojan diseños completamente nuevos y es que en Internet y en la Web muchas veces el modelo que se favorece es el de réplicas o remix (Perkel, 2006), cuyas similitudes con la Web 2.0 son bastante reveladoras (Tabarés-Gutiérrez, 2015, 29 de julio). Además, el carácter artesanal de estas creaciones está mediado digitalmente por los comandos que se desarrollan a la hora de generar los diseños en 3D, el software que controla los componentes, etc. Todos ellos artefactos que responden a unos diseños originales ya preestablecidos y para los cuales el software y el hardware con el que están fabricados siempre disponen de unos parámetros ya predefinidos anteriormente.

\section{¿Hacia la institucionalización de la cultura maker?}

Como hemos visto a lo largo de este artículo, la expansión de la cultura maker a través de innumerables espacios, eventos y diversos colectivos ha atraído la atención de buena parte de los diversos actores institucionales y privados que ven en este fenómeno una manera de apoyar la creatividad, el emprendimiento y la innovación en la ciudadanía. Este interés conlleva a su vez una serie de tensiones a la hora de formalizar 
las actitudes, comportamientos y valores que se encuentran inscritos en el ideario de este movimiento y propicia desencuentros entre los objetivos que se persiguen por los diferentes bandos.

Muchas instituciones ven en este movimiento una manera de apoyar el emprendimiento y el desarrollo de competencias digitales en la ciudadanía y así aliviar la presión que tienen que soportar de cara a la promoción de políticas activas de empleo, y de este modo favorecer la transición hacia un cada vez más competitivo mercado laboral en la sociedad del conocimiento. Estos apoyos financieros pueden generar controversia en los espacios de fabricación digital, ya que muchos de ellos experimentan con diversos modelos de negocio con el objetivo de garantizar su viabilidad económico-financiera, la cual es una de sus problemáticas más recurrentes y acuciantes. Este hecho implica muchas veces aceptar fondos que contravienen sus objetivos y aspiraciones originales y que pueden provocar tensiones entre los diferentes miembros de la comunidad. Lo mismo ocurre con los eventos que se celebran para ensalzar la cultura maker y que son apoyados por empresas privadas, instituciones y otros organismos que pueden tener intereses diferentes a los que tienen este tipo de comunidades.

Y es que la cultura maker es a la vez un síntoma y una transformación del nuevo capitalismo que está alumbrando el fenómeno de la globalización (Lindtner, Bardzell y Bardzell, 2016) y la cada vez mayor importancia de las plataformas digitales en los diferentes ámbitos de la sociedad. Podemos decir que este fenómeno está sucediendo a escala internacional, pero al mismo tiempo dispone de particularidades propias que dependen del contexto donde se circunscribe y que hacen que cada cultura maker nacional, regional o local disponga de especificidades propias de su contexto. No en vano, de lo que estamos hablando en todo momento es de culturas de innovación tecnológica alternativas a las ya establecidas. Lo cual supone una lucha entre los diversos valores e intereses que persiguen y que provocan tensiones en ecosistemas cada vez más complejos y especializados.

La formalización de la cultura maker presenta grandes oportunidades a la hora de promover en la ciudadanía una actitud más crítica para con la tecnología, además de fomentar el desarrollo de competencias digitales y un aprendizaje basado en proyectos colaborativos, pero al mismo tiempo también presenta diversas tensiones con el modo de vida establecido en buena parte de las sociedades occidentales. El motivo no es otro que el uso intensivo de los recursos del planeta por parte de los países desarrollados, que no favorecen modos de consumo más sostenibles y que se acercan a lo que podíamos denominar como economía circular. Esta apuesta por la sostenibilidad es otro de los grandes valores del movimiento maker, que al mismo tiempo refleja las tensiones y transformaciones de una evolución a escala global del sistema capitalista, en busca de una economía más orientada al sector servicios y mediada por las tecnologías digitales. En este sentido podemos concebir el movimiento maker como una especie de manifestación de la contracultura tecnológica que demanda procesos más sostenibles, colaborativos, transparentes, abiertos en la producción y desarrollo de tecnología, y que al mismo tiempo redunden en un beneficio social mayor, más equitativo, justo y compartido.

\section{CONCLUSIONES}

A lo largo del presente texto hemos repasado los orígenes del movimiento maker y su posterior popularización a escala global a través de los innumerables espacios físicos y plataformas digitales donde esta nueva cultura de innovación alternativa se está difundiendo con paso firme. En esta contribución hemos enumerado las diferentes oportunidades que la cultura maker representa a la hora de volver a reenganchar a la ciudadanía con el sector de la fabricación y sus potencialidades para empoderar a los individuos a través del uso de las nuevas tecnologías de uso libre que se han popularizado en la sociedad.

En este sentido es importante destacar que, a pesar de tratarse de un fenómeno emergente, los valores que se circunscriben en él son de una importancia primordial de cara a la transición de la sociedad hacia entornos postindustriales, en los que el papel de la innovación, la experimentación, el aprendizaje social y el acceso al conocimiento irán cobrando cada vez más importancia, al mismo tiempo que irán relegando otros valores actualmente presentes en estructuras provenientes de la etapa industrial. Por ello, la irrupción y popularización del movimiento maker es un anticipo de las nuevas sensibilidades que están forjando el uso de las nuevas tecnologías que devienen de la revolución digital, y simultáneamente una expresión por una parte de la sociedad de la necesidad de repensar nuestras estructuras actuales de desarrollo productivo y tecnológico.

Por este motivo es importante prestar atención a este tipo de movimientos emergentes, ya que suponen una llamada de atención sobre ciertos aspectos de la sociedad a los que se critica de manera activa y 
a los que se proponen rutas alternativas, con el fin de facilitar las transiciones y transformaciones que urge afrontar a corto y medio plazo. En este trabajo se ha prestado especial atención a la importancia que posee la cultura tecnológica en el movimiento maker a la hora de crear rutas alternativas en el desarrollo tecnológico y permitir a la ciudadanía su empoderamiento a través de la apropiación social de la tecnología, pero al mismo tiempo se ha tratado de poner de relieve la necesidad de promover en la sociedad nuevos valores que sirvan para afianzar su transición hacia un nuevo tipo de economía.

En este texto se ha tratado de prestar especial atención a ciertos valores que propicia esta nueva ética maker y cómo son considerados como fundamentales a la hora de promover una cultura de la innovación en la sociedad, la cual será crucial de cara al asentamiento de la tan ansiada economía del conocimiento. Este hecho se ve favorecido por la cada vez mayor digitalización de industrias y sectores enteros, que propician la orientación de la economía hacia el sector tercero, hacia la generación de servicios de valor añadido. En este sentido, es necesario que se desarrollen nuevas culturas de la innovación para propiciar nuevas formas de valor que produzcan prosperidad y riqueza, y para generar en la sociedad nuevos valores respecto a la innovación.

Por ello el movimiento maker puede ser una fuerza regeneradora que, convenientemente apoyada y canalizada por las instituciones y otros agentes, puede proveer numerosas oportunidades de renovación en sectores como la educación, la fabricación y la artesanía para crear nuevas estructuras que sean capaces de albergar las nuevas sensibilidades necesarias para facilitar la transición hacia una economía postindustrial.

\section{AGRADECIMIENTOS}

Esta publicación se inscribe en el marco del programa de apoyo a Centros Tecnológicos y sus Corporaciones y Alianzas Tecnológicas Emaitek del gobierno vasco, y del proyecto OPENMAKER, que ha recibido financiación de la Comisión Europea a través del programa Horizonte 2020 bajo el número de contrato 687941 .

\section{NOTAS}

1. P2P es el acrónimo de Peer to Peer en inglés.

2. Más información sobre esta Colonia Eco-Industrial Post-capitalista en https://calafou.org/es/content/ acerca-de

3. Esta plataforma ha tenido una gran influencia de The Whole Earth Catalog (http://www.wholeearth.com/index.php) que fue una importante publicación dirigida por Stewart Brand. La dirección web de Thingiverse es http:// www.thingiverse.com

4. Se puede consultar la página de la Wikipedia en castellano para conocer más información sobre la iniciativa Fab Lab en https:// es.wikipedia.org/wiki/Fab_lab

5. En este enlace se puede consultar la cifra actualizada en todo momento https://www.fablabs.io/labs

6. La referencia se puede consultar en el siguiente enlace: https:// wiki.hackerspaces.org/List_of_Hacker_spaces

7. Se puede consultar más información relativa a la iniciativa TechShop en la página que dedica la Wikipedia en inglés https://en.wikipedia. org/wiki/TechShop

8. Una breve explicación de esta quiebra puede encontrarse en el siguiente enlace por parte de su fundador, David Woods: https:// makezine.com/2017/11/15/techshop-closes-doors-files-bankruptcy/

9. Una pequeña reseña de la historia del evento Maker Faire se puede encontrar en su propia página web: https://makerfaire.com/makerfairehistory/

10. Puede consultarse en el siguiente enlace: https://obamawhitehouse. archives.gov/blog/2014/06/18/president-obama-white-house-makerfaire-today-s-diy-tomorrow-s-madeamerica

11. Los detalles del programa se pueden consultar en la siguiente dirección https://nationofmakers.us/

12. Se puede encontrar más información en: https://www.usnews. com/news/maker-cities/articles/2017-05-23/maker-cities-ifyou-can-imagine-it-you-can-build-it
13. Más información sobre este programa de la Comunidad Europea en el siguiente enlace: https://ec.europa. eu/programmes/horizon2020/en/ h2020-section/science-and-society

14. https://make-it.io/

15. https://making-sense.eu/

16. http://openmaker.eu/

17. http://odmplatform.eu/

18. http://www.makerfairerome.eu/en/

19. https://makerstown.eu/

20. https://ajuntament.barcelona.cat/ digital/ca/apoderament-digital/ educacio-i-capacitacio-digital/ateneus-de-fabricacio

21. Disponible en: http://www.elperiodico.com/es/noticias/distritos/ lateneu-fabricacio-siembra-tecnologia-digital-2624769

22. Más información sobre la iniciativa en el siguiente enlace: https:// www.tknika.eus/cont/proyectos/ ikaslab/\#

23. Más información en: https://www. spri.eus/es/basque-industry/ 


\section{BIBLIOGRAFÍA}

A third industrial revolution (2012, 12 de abril). The Economist. [En línea]. Disponible en: http://www.economist. com/node/21552901

Anderson, C. (2012). Makers: the new industrial revolution. Random House.

Atkinson, P. (2006). Do It Yourself: Democracy and Design. Journal of Design History, 19 (1), pp. 1-10. https://doi. org/10.1093/jdh/epk001

Barbrook, R. y Cameron, A. (1996). The Californian ideology. Science as Culture, 6 (1), pp. 44-72. https://doi. org/10.1080/09505439609526455

Benkler, Y. (2006). The Wealth of Networks: How Social Production Transforms Markets and Freedom. New Haven and London: Yale University Press.

Birtchnell, T. y Urry, J. (2013). Fabricating Futures and the Movement of Objects. Mobilities, 8 (3), pp. 388-405. https://doi.org/10.1080/17450101.2 012.745697

Chesbrough, H. (2003). Open innovation: The new imperative for creating and profiting from technology. Harvard, MA: Harvard Business Press.

Cruz, J., Herrington, S. y Rodríguez, B. (2014). Farmbot. [En línea]. Disponible en: http://digitalcommons.calpoly.edu/mesp/241

Dafermos, G. (2015). Transforming the productive base of the economy through the open design commons and distributed manufacturing. The Journal of Peer Production, 7. Disponible en: http://peerproduction. net/issues/issue-7-policies-for-thecommons/peer-reviewed-papers/ distributed-manufacturing/

Dafermos, G. y Söderberg, J. (2009) The hacker movement as a continuation of labour struggle. Capital \& Class, 33 (1), pp. 53-73. https://doi. org/10.1177/030981680909700104

Dougherty, D. (2012). The Maker Movement. Innovations: Technology, Governance, Globalization, 7 (3), pp. 11-14. https://doi.org/10.1162/ INOV_a_00135

Echeverría, J. (2008). El manual de Oslo y la innovación social. Arbor, 184 (732), pp. 609-618. Disponible en: http://arbor.revistas.csic.es/index.php/arbor/ article/viewArticle/210
Farrell, J. y Klemperer, P. (2007). Coordination and lock-in: Competition with switching costs and network effects. En: Handbook of Industrial Organization, vol. 3, pp. 1967-2072. https://doi. org/10.1016/S1573-448X(06)03031-7

Fox, S. (2014). Third Wave Do-It-Yourself (DIY): Potential for prosumption, innovation, and entrepreneurship by local populations in regions without industrial manufacturing infrastructure. Technology in Society, 39, pp. 18-30. https://doi.org/10.1016/j.techsoc.2014.07.001

García-Sáez, C. (2016). (Casi) Todo por hacer. Una mirada social y educativa sobre los Fab Labs y el movimiento maker. Madrid: Fundación Orange.

Gershenfeld, N. (2005). Fab: The coming revolution on your desktop - From personal computers to personal fabrication. New York: Basic Books.

Halverson, E. R. y Sheridan, K. (2014). The Maker Movement in Education. Harvard Educational Review, 84 (4), pp. 495-504. https://doi.org/10.17763/ haer.84.4.34j1g68140382063

Haring, K. (2008). Ham Radio's Technical Culture. Cambridge, Mass: The MIT Press.

Hatch, M. (2013). The maker movement manifesto: rules for innovation in the new world of crafters, hackers, and tinkerers. London: McGraw Hill.

Himanen, P. (2002). La ética del hacker y el espíritu de la era de la información. Barcelona: Destino.

Hippel, E. von (2005). Democratizing Innovation. Cambridge, MA: The MIT Press. Disponible en: http://web.mit. edu/evhippel/www/democ1.htm

Kneese, T., Rosenblat, A. y Boyd, D. (2014). Technologically Mediated Artisanal Production. Future of Work. Data \& Society Working Paper. [En línea]. https:// doi.org/10.2139/ssrn.2536613

Kostakis, V., Niaros, V., Dafermos, G. y Bauwens, M. (2015). Design global, manufacture local: Exploring the contours of an emerging productive model. Futures, 73, pp. 126-135. https://doi.org/10.1016/j.futures.2015.09.001

Kostakis, V. y Papachristou, M. (2014). Commons-based peer production and digital fabrication: The case of a RepRap-based, Lego-built 3D printing-milling machine. Telematics and Informatics, 31 (3), pp. 434-443. https://doi.org/10.1016/j. tele.2013.09.006

Kuznetsov, S. y Paulos, E. (2010). Rise of the Expert Amateur: DIY Projects, Communities, and Cultures. Proceedings of the 6th Nordic Conference on Human-Computer Interaction: Extending Boundaries. ACM, pp. 295-304. https://doi. org/10.1145/1868914.1868950

Leonard, A. (2013, 26 de julio). The Maker economy can't fix everything. [En línea]. Disponible en: http://www. salon.com/2013/07/26/the maker economy_cant_fix_everything/

Lindtner, S. (2014). Hackerspaces and the Internet of Things in China: How makers are reinventing industrial production, innovation, and the self. China Information, 28, pp. 145-167. https:// doi.org/10.1177/0920203X14529881

Lindtner, S. (2015). Hacking with Chinese Characteristics: The Promises of the Maker Movement against China's Manufacturing Culture. Science, Technology \& Human Values, 40 (5), pp. 854-879. https://doi. org/10.1177/0162243915590861

Lindtner, S., Bardzell, S. y Bardzell, J. (2016). Reconstituting the Utopian Vision of Making. Proceedings of the 2016 CHI Conference on Human Factors in Computing Systems - CHI '16. ACM, pp. 1390-1402. https://doi. org/10.1145/2858036.2858506

Lindtner, S. y Li, D. (2012). Created in China: The Makings of China's Hackerspace Community. Interactions, 19, pp. 18-22. https://doi. org/10.1145/2377783.2377789

Lipson, H. y Kurman, M. (2010). Factory @ Home: The Emerging Economy of Personal Fabrication. [En línea]. Disponible en: http://citeseerx.ist.psu. edu/viewdoc/download?doi=10.1.1. 469.3463\&rep=rep1\&type=pdf

Locoro, A., Ravarini, A., Cabitza, F. y Mari, L. (2017). Is making the new knowing ? Tangible and intangible knowledge artifacts in DIDIY. En Proceedings of the 25th European Conference on Information Systems (ECIS) (Vol. 2017). Guimaraes: AIS Electronic Library (AISeL). Disponible en: 
https://aisel.aisnet.org/cgi/viewcontent.cgi ?article $=1018 \&$ context $=e c$ is2017_rp

Martin, L. (2015). The Promise of the Maker Movement for Education. Journal of Pre-College Engineering Education Research (J-PEER), 5 (1). https://doi.org/10.7771/21579288.1099

Morozov, E. (2014, 23 de enero). Making It. The New Yorker. Disponible en: http://www.newyorker.com/magazine/2014/01/13/making-it-2

Mosterín, J. (1993). Filosofía de la cultura. Homo. Madrid: Alianza.

Mota, C. (2011). The Rise of Personal Fabrication. Proceedings of the 8th ACM conference on Creativity and cognition. ACM, pp. 279-288. https://doi. org/10.1145/2069618.2069665

Niaros, V., Kostakis, V. y Drechsler, W. (2017). Making (in) the Smart City: The Emergence of Makerspaces. Telematics and Informatics, 34 (7), pp. 1143-1152. Disponible en: https:// doi.org/10.1016/j.tele.2017.05.004

Olivé, L. (2005). La cultura científica y tecnológica en el tránsito a la sociedad del conocimiento. Revista de la Educación Superior, XXXIV (4) (136), pp. 49-63. Disponible en: http://www. redalyc.org/html/604/60413604/

Perkel, D. (2006). Copy and paste literacy? Literacy practices in the production of a MySpace profile. En: Informal Learning and Digital Media: Constructions, Contexts, Consequences, pp. 21-23. Disponible en: http://people.ischool. berkeley.edu/ dperkel/writing/perkel_copypasteliteracyDRAFT_August2007.pdf
Quintanilla, M. Á. (1997). El concepto de progreso tecnológico. Arbor, CLVII (620), pp. 370-390. https://doi. org/10.3989/arbor.1997.i620.1822

Quintanilla, M. Á. (1998). Técnica y cultura. Teorema, XVII (3), pp. 49-69. Disponible en: http://www.oei.es/ salactsi/teorema03.htm

Quintanilla, M. Á. (2005). Tecnología: Un enfoque filosófico y otros ensayos de filosofía de la tecnología. Mexico D.F.: Fondo de Cultura Económica.

Ratto, M. y Ree, R. (2012). Materializing information: $3 \mathrm{D}$ printing and social change. First Monday, 17 (7), pp. 1-25. https://doi.org/10.5210/fm.v17i7.3968

Rheingold, H. (1996). La comunidad virtual: una sociedad $\sin$ fronteras. Gedisa.

Rogers, E. M. (1962). Diffusion of Innovations. New York: Free Press.

Sivek, S. C. (2011). "We Need a Showing of All Hands": Technological Utopianism in MAKE Magazine. Journal of Communication Inquiry, 35 (3), pp. 187-209. https://doi. org/10.1177/0196859911410317

Smith, A., Hielscher, S., Dickel, S., Söderberg, J. y Oost, E. van. (2013). Grassroots digital fabrication and makerspaces: reconfiguring, relocating and recalibrating innovation? SPRU-Science and Technology Policy Research No. 2013-02. [En línea]. Disponible en: https://www. ioew.de/fileadmin/user_upload/ BILDER_und_Downloaddateien/Publikationen/2014/Smith_Hielscher_ Dickel_S\%C3\%B6derberg_Oost_Grassroots.pdf

Tabarés-Gutiérrez, R. (2015, July 29). La Belleza del Código: Influencia de la Web 2.0, los medios sociales y los contenidos multimedia en el desarroIlo de HTML5. [Tesis doctoral inédita]. Universidad de Salamanca: Salamanca. Disponible en: http://dsp.tecnalia. com/handle/11556/190

Tabarés-Gutiérrez, R. (2016). Approaching maker's phenomenon. Interaction Design and Architecture(s), 30, pp. 19-29.

Tabarés-Gutiérrez, R., Bartolomé, T., Martelloni, L., Marmo, D., Amicis, L. de, Binenti, S. [...] y Billy, L. (2018). Exploring the Emergent Open Manufacturing Industry-OPENMAKER White Paper v1.0. Disponible en: http://openmaker.eu/ wp-content/uploads/2018/02/D4.3White-paper-version-1.0.pdf

Tabarés-Gutiérrez, R., Sopelana, A., García, J. y Moreno-Valdés, M. T. (2016). Social Manufacturing: Towards the popularization of personalized fabrication. Science and Technology by Other Means - Exploring collectives, spaces and futures EASST / 4S 2016. Barcelona. Disponible en: https:// www.researchgate.net/publication/311739460_Social_Manufacturing_Towards_the_popularization of_personalized_fabrication

Toboso-Martín, M. (2014). Perspectiva axiológica en la apropiación social de tecnologías. Revista Iberoamericana de Ciencia, Tecnología Y Sociedad, 9(25), 1-18.

Wenger, E. (1999). Communities of practice: Learning, meaning, and identity. Cambridge University Press. 